\title{
Cobertura e fatores associados com a realização do exame Papanicolaou em municipio do Sul do Brasil
}

\author{
Coverage and factors associated with Papanicolaou testing in a city of Southern Brazil \\ Daniela Wosiack da Silva ${ }^{1}$, Selma Maffei de Andrade ${ }^{2}$, Darli Antonio Soares², \\ Barbara Turini², Camilla Alexsandra Schneck ${ }^{3}$, Maria Lúcia da Silva Lopes ${ }^{4}$
}

\section{RESUMO}

Objetivo: verificar a cobertura e fatores associados à adesão ao exame Papanicolaou em áreas de Londrina (PR). Métodos: estudo transversal em microáreas de cinco Unidades Básicas de Saúde (UBS), em 2004. Em cada UBS, foram selecionadas uma ou duas microáreas e relacionadas, por consulta ao Sistema de Informação da Atenção Básica, todas as mulheres residentes de 20 a 59 anos, as quais foram visitadas e entrevistadas. Foram consideradas como com exame atualizado aquelas submetidas à coleta nos três anos anteriores à entrevista, e em atraso as demais. Foi estudada a associação de alguns fatores em relação à situação do exame. Para o processamento e análise dos dados usou-se o programa Epi-Info 6.04d. Resultados: participaram do estudo 513 mulheres. A cobertura geral do exame foi de $80,7 \%$, variando de 71,5 a $88,4 \%$ nas cinco áreas. Foi significativamente maior a proporção de exame em atraso $(p<0,05)$ entre mulheres que trabalhavam somente em casa $(22,4 \%$ em comparação a $14,3 \%$ das que trabalhavam fora) e entre as que pertenciam às classes econômicas $\mathrm{D} / \mathrm{E}(24,9 \%)$, comparativamente às classes $\mathrm{C}(17,5 \%)$ e A/B $(8,3 \%)$. Observou-se maior proporção de desconhecimento da data de realização do próximo exame $(\mathrm{p}<0,01)$ entre as que se submeteram à coleta na UBS $(14,7 \%)$, em comparação àquelas de serviços privados ou conveniados (5,8\%). Conclusão: houve boa cobertura geral do exame nas áreas das UBS pesquisadas, porém são necessárias ações para maior adesão das mulheres em atraso com o exame, em especial daquelas com piores condições financeiras e que trabalham exclusivamente em casa.

PALAVRAS-CHAVE: Serviços de saúde; Saúde pública; Neoplasias de colo uterino; Esfregaço vaginal; Citologia; Brasil

\section{ABSTRACT}

Purpose: to verify the coverage and factors associated with Papanicolaou (Pap) testing in Londrina (PR), Brazil. Methods: this is a cross-sectional study, carried out in 2004, in microareas of five Basic Health Units (BHU) of Londrina. One or two microareas from each BHU were selected and a list of all women aged 20-59 years resident in these places, was made through search in the Basic Attention Information System, the women being then visited and interviewed. Those with a Pap test in the last three years were considered as having an updated examination, and the remaining as delayed. The association of some factors with the examination situation was investigated. Data analysis was performed using Epi-Info 6.04d. Results: Pap smear coverage among the 513 participants of the study was $80.7 \%$, ranging from $71.5 \%$ to $88.4 \%$. Delay in taking the test was higher $(\mathrm{p}<0.05)$ among women who worked only at home $(22.4 \%$ as compared with $14.3 \%$ among those who worked outside), and among those who belonged to $\mathrm{D} / \mathrm{E}$ social classes $(24.9 \%)$ as compared to $\mathrm{C}(17.5 \%)$ and $\mathrm{A} / \mathrm{B}(8.3 \%)$ classes. The proportion who ignored the next test date was higher $(\mathrm{p}<0.01)$ among those who had the last Papanicolaou testing at a BHU (14.7\%), as compared to those who had been attended privately or by a health insurance company (5.8\%). Conclusion: the coverage of Pap smear in the studied areas can be considered satisfactory, although there is a need of improving compliance with Pap test, mainly among women who are the poorest and who work only at home.

KEYWORDS: Health services; Public health; Cervix neoplasms; Vaginal smears; Cytology; Brazil

Universidade Estadual de Londrina/Centro de Ciências da Saúde.

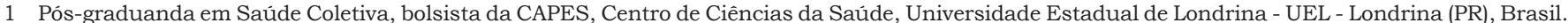

2 Docentes do Departamento de Saúde Coletiva, Centro de Ciências da Saúde, Universidade Estadual de Londrina - UEL - Londrina (PR), Brasil.

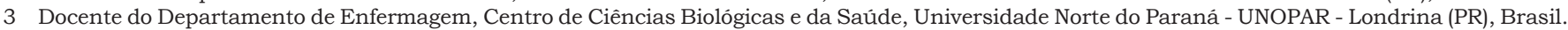

4 Docente do Departamento de Enfermagem, Centro Universitário Filadélfia de Londrina - UNIFIL - Londrina (PR), Brasil.

Correspondência: Daniela Wosiack da Silva.

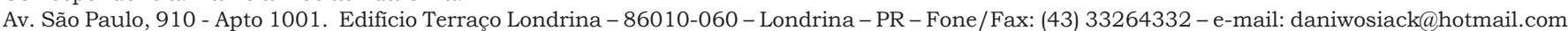


Introdução

O câncer de colo uterino é o segundo mais comum entre mulheres no mundo. A grande maioria dos casos novos ocorre em países em desenvolvimento, onde, em algumas regiões, é o tipo de câncer mais comum na população feminina ${ }^{1}$. Atualmente, estima-se que ocorram cerca de $235 \mathrm{mil}$ mortes por câncer de colo uterino por ano no mundo, sendo 218 mil em nações consideradas pobres ou emergentes ${ }^{2}$.

No Brasil, os dados mais recentes sobre mortalidade divulgados pelo Ministério da Saúde informam 4.202 mortes por carcinoma de colo uterino em 2003, representando a quarta principal causa de morte por câncer entre mulheres ${ }^{3}$. Esse número, embora ainda elevado, possivelmente está subestimado, considerando que casos de morte por câncer de colo de útero podem ser informados, na declaração de óbito, como de porção não especificada de útero ${ }^{4}$, ou, ainda, por outras causas.

Em termos de incidência da doença, estudos nacionais de base populacional são praticamente inexistentes, porém as estimativas do Instituto Nacional de Câncer (INCA) ${ }^{5}$ apontam taxas, em 2006, que variam de 8,4 (Paraíba) até 30,9 (Rio Grande do Sul) por 100.000 mulheres. O estado do Paraná, com uma taxa de 27,5 casos novos estimados por 100.000 mulheres, em 2006, é o terceiro com maior taxa estimada no Brasil, sendo esta inferior, apenas, às dos estados do Rio Grande do Sul e Amazonas ${ }^{5}$.

O principal fator associado com a ocorrência de câncer de colo uterino é a infecção pelo papilomavirus humano (HPV) ${ }^{2}$. São co-fatores para o desenvolvimento deste tipo de neoplasia a multiplicidade de parceiros sexuais, história de infecções sexualmente transmitidas e tabagismo ${ }^{6}$. Estudo que analisou a mortalidade mundial por diversos tipos de câncer destaca que o tabagismo e a relação sexual sem preservativo são responsáveis, respectivamente, por 2 e 100\% das mortes por câncer de colo uterino em todo o mundo, sendo a transmissão sexual do HPV a principal causa deste tipo de câncer em mulheres de países pobres ou em desenvolvimento ${ }^{2}$.

Quando detectado precocemente, o câncer de colo de útero tem possibilidade de cura em praticamente todos os casos. O exame de Papanicolaou (citologia oncótica) tem sido o método adotado para detecção desse tipo de câncer já há várias décadas na maioria dos países, com diferentes estratégias de organização do programa de rastreamento, em especial relacionadas à periodicidade do exame e à faixa etária alvo, com variados ní- veis de sucesso na redução da morbimortalida$\mathrm{de}^{4,7,8}$. Na Suécia, um estudo ${ }^{7}$ revelou que mudanças no programa de rastreamento do câncer de colo uterino aumentou a cobertura na faixa etária-alvo em $8,4 \%$ e reduziu a incidência de câncer invasivo de colo uterino em $22 \%$ no período de 1992 a 2000. Nesse país, passou-se de atendimento exclusivo à demanda espontânea para enfoque em mulheres de 25 a 69 anos que não haviam se submetido ao teste nos últimos três anos, por meio de convocação por cartas ${ }^{7}$. De forma similar, no Chile, alterações foram introduzidas no programa em 1987, passando de exame anual, em mulheres que espontaneamente procuravam clínicas de atendimento, para exames trienais em mulheres de 25 a 64 anos $^{8}$. Como conseqüência, observouse aumento da proporção de mulheres desta faixa etária que haviam se submetido ao exame Papanicolaou nos últimos três anos (de 51\% em 1990 para 66\% em 1996), com redução da mortalidade por este tipo de câncer: de 11,1 por 100.000 mulheres em 1986 para 6,8 em 20018. No Brasil, o Ministério da Saúde preconiza a realização anual do exame Papanicolaou (citologia oncótica) em mulheres de 25 a 60 anos ou nas sexualmente ativas e, após dois exames anuais consecutivos negativos, a cada três anos ${ }^{9}$.

Apesar da maior ocorrência de casos de câncer de colo uterino nos países pobres ou em desenvolvimento, poucos são os estudos realizados sobre a cobertura do exame Papanicolaou nesses países, incluindo o Brasil ${ }^{10,11}$. Ainda mais escassos são os estudos realizados sobre os fatores associados à realização ou não do exame, em especial os que procuram identificar os motivos relatados pelas próprias mulheres para a prática do exame $^{12}$. Em publicação recente que analisou a produção científica brasileira sobre o assunto, Martins et al. ${ }^{11}$ destacam que há poucas investigações no País, e estas se concentram especialmente em cidades dos estados de São Paulo e do Rio Grande do Sul. Há, no entanto, evidências de que, nos últimos anos, vem aumentando o percentual de mulheres que se submetem ao exa$\mathrm{me}^{11}$, possivelmente em razão da descentralização dos serviços de saúde ocorrida nas últimas décadas no Brasil.

Monitoramento e avaliação do programa de detecção precoce do câncer de colo uterino são essenciais para efetivos e eficientes planejamento e organização dos serviços de saúde, especialmente em países com recursos limitados ${ }^{13}$. Estudos transversais realizados periodicamente podem suprir as necessidades de monitoramento e de informação dos serviços de saúde a respeito das coberturas alcançadas, dos principais problemas 
a serem contornados, dos motivos de realização ou não do exame de citologia oncótica, entre outros ligados à estrutura dos serviços de saúde e do processo da atenção.

Nos últimos anos, vem crescendo a cobertura de serviços de saúde no Brasil por meio da estratégia Saúde da Família, a qual pressupõe uma equipe minima composta por médico, enfermeiro, auxiliares de enfermagem e agentes comunitários de saúde, e um modelo que visa reverter o da assistência centrada na doença, deslocando o foco também para a promoção da saúde e a prevenção de doenças. Tal estratégia tem se mostrado importante na redução das iniqüidades no acesso e utilização dos serviços de saúde ${ }^{14}$. No município de Londrina, estado do Paraná, tal estratégia foi implantada em 2001, e atualmente atinge praticamente a totalidade da população residente em áreas urbanas periféricas, de baixo poder aquisitivo. Nesta cidade, como em outros locais do Brasil, há carência de estudos que avaliem, entre mulheres atendidas por serviços de saúde que utilizam a estratégia Saúde da Família, a cobertura do exame Papanicolaou e os fatores que dificultam ou favorecem sua adesão ao exame, o que poderia contribuir para a reorganização dos serviços visando contornar possiveis barreiras de acesso ao exame.

Diante desse contexto, o objetivo deste estudo foi o de verificar a cobertura do exame de detecção do câncer de colo de útero (Papanicolaou) e levantar fatores associados à sua realização em Londrina, município de médio porte do estado do Paraná, Região Sul do Brasil, tendo em vista a importância do tema para a saúde da mulher e a recente reorganização do processo de trabalho local com a implantação da estratégia Saúde da Família.

\section{Métodos}

Trata-se de estudo transversal, realizado em microáreas de abrangência de cinco das 40 Unidades Básicas de Saúde (UBS) da zona urbana do município de Londrina, estado do Paraná, no período de setembro a outubro de 2004. O município conta, atualmente, com população projetada em cerca de 490 mil habitantes, e apresenta boa distribuição geográfica das UBS (próximas à população). Todas as áreas estudadas adotam a estratégia da Saúde da Família, o que permite contato direto e freqüente com a população.

Estas unidades de saúde foram definidas por constituírem campos de ensino para alunos do módulo de "Práticas de Interação Ensino-Serviço-
Comunidade" (PIN-2) das segundas séries dos cursos de Medicina e Enfermagem da Universidade Estadual de Londrina. Este módulo tem como objetivo, entre outros, propiciar aos alunos a participação e aprendizado nas diversas etapas de uma pesquisa científica, em contato direto com os serviços básicos de atenção à saúde e a população ${ }^{15}$.

Em cada UBS, foram selecionadas uma ou duas microáreas e relacionadas todas as mulheres de 20 a 59 anos residentes nesses locais, por intermédio de consulta aos registros do Sistema de Informação da Atenção Básica (SIAB). A seleção das microáreas foi realizada da seguinte forma: nas três unidades que apresentavam homogeneidade quanto à composição socioeconômica, as microáreas incluídas no estudo foram selecionadas por sorteio; nas duas unidades que apresentavam heterogeneidade socioeconômica, foram selecionadas as de maior risco social. A categorização de risco social é prática comum realizada pelas equipes de saúde em oficinas de territorialização em toda a rede de saúde de Londrina, sendo estimado a partir de informações sobre condições de habitação e saneamento, escolaridade e poder aquisitivo da população ${ }^{16}$.

Foram realizadas, então, visitas domiciliares e entrevistas, por alunos do Módulo PIN-2, treinados e supervisionados por docentes instrutores. Para registro dos dados das entrevistas, foi utilizado formulário padronizado, contendo questões fechadas e abertas. As mulheres foram entrevistadas em suas próprias residências, após explanação dos objetivos da pesquisa e de leitura e assinatura de um termo de consentimento livre e esclarecido. Foram excluídas da amostra, após as entrevistas, as mulheres com menos de 25 anos não sexualmente ativas e as que haviam sido submetidas a histerectomia.

A variável dependente considerada no estudo foi a situação das mulheres quanto a terem se submetido ao exame Papanicolaou: nunca se submeteram, foram submetidas há menos de um ano, entre um e três anos, há três anos ou mais ou não se lembra. Para efeitos da análise bivariada, as categorias referentes à variável dependente foram agrupadas em: submeteu-se ao exame há menos de três anos (situação considerada como exame atualizado) e não se submeteu ao exame há menos de três anos (o exame foi coletado há três anos ou mais, nunca foi coletado ou não se lembra), situação esta considerada como exame em atraso.

Como variáveis independentes foram consideradas a idade da entrevistada, o estado conjugal, a cor da pele, o trabalho fora de casa, a classe econômica, a utilização habitual da UBS, o local de 
coleta do último exame e o tempo necessário para se locomover de sua residência até a UBS (em minutos). A cor da pele das participantes do estudo foi definida de acordo com a percepção dos entrevistadores. Constavam no formulário as seguintes categorias: cor branca, cor parda/negra e amarela. A classe econômica foi estimada conforme a metodologia da Associação Brasileira de Empresas de Pesquisa - $\mathrm{ABEP}^{17}$, baseando-se na posse de bens e na escolaridade do chefe da família. Segundo critérios adotados pela ABEP, maiores pontuações são obtidas quando a família tem a propriedade de bens duráveis como televisão a cores, carro, máquina de lavar, aspirador de pó e outros. Contam pontos, também, o número de banheiros na residência e a disponibilidade de empregada mensalista, além do grau de instrução do chefe da família. As classes econômicas definidas por essa metodologia são transformadas em letras de A (classe com maior poder de consumo) até $\mathrm{E}$ (menor poder). O estado conjugal admitia as seguintes categorias: com companheiro (casada ou que mantinha relação estável) e sem companheiro.

As variáveis independentes, na análise bivariada, foram agrupadas da seguinte forma: idade (20 a 39 ou 40 a 59 anos), trabalho fora de casa (sim ou não), utilização habitual da UBS (sim ou não), estado conjugal (com companheiro ou sem companheiro), local de realização do último exame (UBS ou outro local), cor da pele (cor branca/ amarela ou cor parda/negra), classe econômica (classes econômicas A/B, classe C ou classes D/ E) e o tempo necessário para se locomover de sua residência até a UBS (variável esta considerada como numérica, sem agrupamentos).

Foram investigados ainda os motivos para a adesão ou não ao Papanicolaou entre as mulheres que estavam com o exame atualizado e entre as com o exame em atraso, respectivamente. Constavam no questionário os seguintes motivos para as que compareceram para coleta do exame nos últimos três anos: recomendação do médico ou enfermeiro, queixa ginecológica, recomendação de amigos ou parentes, rotina do programa e outros motivos (questão aberta). Para o não-comparecimento para coleta constavam os seguintes: falta de tempo, medo do resultado, vergonha, desinformação, descrença na UBS, proibição do marido/companheiro, dificuldade no agendamento e outros motivos (questão aberta). Tanto para a adesão como para a não-adesão ao Papanicolaou admitiu-se mais de uma resposta.

Também foi investigado o conhecimento das entrevistadas que se encontravam com o exame atualizado sobre a data de agendamento do próximo preventivo de acordo com local de coleta do úl- timo exame (UBS ou locais que prestam atendimento privado ou por convênios médicos).

Para processamento e análise dos dados foi utilizado o programa Epi-Info versão $6.04 \mathrm{~d}^{18}$. O teste do $\chi^{2}$ de Pearson foi usado para verificar associações entre as variáveis independentes e a situação relativa ao exame Papanicolaou nos últimos três anos, sendo considerado um nível de significância de 5\%, com um ou dois graus de liberdade, conforme categorização dessas variáveis independentes ${ }^{19}$. Também foi aplicada a análise estratificada de Mantel-Haenszel para verificar possivel interação entre variáveis associadas ao desfecho analisado (exame de citologia oncótica considerado atualizado ou não nos últimos três anos). O teste de Kruskal-Wallis foi utilizado para comparação de médias, considerando a distribuição não gaussiana das freqüências das variáveis analisadas.

O projeto de pesquisa foi aprovado pelo Comitê de Ética em Pesquisa da Universidade Estadual de Londrina, conforme parecer CEP 235/04.

\section{Resultados}

Foram entrevistadas 518 mulheres. Destas, foram excluídas quatro histerectomizadas e uma mulher não sexualmente ativa com menos de 25 anos identificadas após a realização da entrevista. A cobertura geral do exame entre as $513 \mathrm{mu}-$ lheres participantes do estudo nas microáreas das cinco unidades básicas de saúde pesquisadas foi de $80,7 \%$, com variação de $71,5 \%$ a $88,4 \%$.

Constatou-se que 41 mulheres participantes do estudo $(8,0 \%)$ referiram nunca ter se submetido ao exame Papanicolaou na vida, $48(9,4 \%)$ relataram que compareceram para coleta há três anos ou mais, $10(1,9 \%)$ não se lembraram de sua coleta, $138(26,9 \%)$ referiram que haviam se submetido ao exame entre um e três anos e 276 $(53,8 \%)$ há menos de um ano.

Os principais motivos citados para a adesão ao Papanicolaou entre as 414 mulheres que se encontravam com o exame atualizado (exame coletado há menos de três anos) foram: rotina do programa oferecido pela UBS (46,9\%), recomendação médica $(25,8 \%)$ e queixas ginecológicas $(14 \%)$. Desse grupo, 66,3\% haviam se submetido ao último exame na UBS da área e as demais em locais que prestam atendimento privado ou por convênios médicos (excluído um caso que não informou o local de realização do exame). Observou-se associação significativa entre o local de coleta do último exame e a classe econômica, sendo menor a 
proporção de utilização da UBS entre mulheres das classes econômicas de maior poder aquisitivo: $35,3 \%$ das classes A/B, 69,5\% da classe C e 78,5\% das classes $\mathrm{D} / \mathrm{E}(\mathrm{p}<0,001)$.

Considerando-se ainda apenas as mulheres com exame atualizado, notou-se diferença a respeito do conhecimento quanto à data aproximada de coleta do próximo exame, sendo maior a proporção de relato de desconhecimento entre as mulheres que referiram o último exame na UBS $(14,7 \%)$ quando comparadas às mulheres que relataram o último exame em locais que prestam atendimento privado ou por convênios médicos $(5,8 \%)(p<0,01)$. Esta associação entre local de coleta do último exame e desconhecimento da data aproximada do próximo se manteve, mesmo após controle da variável classe econômica.

Entre as 99 mulheres que estavam com a situação em atraso (exame coletado há três anos ou mais ou que nunca compareceram para coleta, ou que não se lembravam), os principais motivos relatados para a não adesão ao exame foram vergonha $(24,2 \%)$, desinformação $(19,2 \%)$, falta de interesse $(13,1 \%)$, dificuldade para o agendamento do exame $(8,1 \%)$, o fato de não gostar do(a) médico(a) da UBS $(6,1 \%)$ e nunca ter tido queixa ginecológica $(4 \%)$.

Das 460 mulheres que informaram o tempo necessário para se locomover de sua residência até a UBS, 95\% relataram um tempo de até 30 minutos, com média e mediana, respectivamente, de 16 e 15 minutos. Comparando os tempos necessários para deslocamento entre residência e UBS, não se observou diferença significativa (teste Kruskal-Wallis; $p=0,199$ ) entre as mulheres com exame em atraso ou atualizado.

Observaram-se maiores proporções de atraso entre as mulheres de cor parda/negra $(22,4 \%)$, de classes econômicas menos favorecidas (D/ $\mathrm{E}=24,9 \% ; \mathrm{C}=17,5 \%)$, sem companheiro $(24,3 \%)$ e que não trabalham fora de casa $(22,4 \%)$ (Tabela 1). Porém, somente o tipo de trabalho e a classe econômica estiveram associados, de forma significativa, com o não comparecimento para coleta do exame Papanicolaou nos últimos três anos, sendo maiores as proporções entre as mulheres que não trabalhavam fora e entre as que pertenciam às classes econômicas $\mathrm{D}$ e $\mathrm{E}$, seguidas pelas da classe $C(p<0,05)$. A análise estratificada de Mantel-Haenszel mostrou não haver interação entre estas duas variáveis (trabalho e classe econômica), sendo, portanto, ambas consideradas associadas de forma independente com a não-adesão ao exame de citologia oncótica nos últimos três anos.
Tabela 1 - Características sociodemográficas e utilização da Unidade Básica de Saúde (UBS) de acordo com a situação do exame Papanicolaou nos últimos três anos, Londrina, 2004.

\begin{tabular}{|c|c|c|c|c|c|}
\hline \multirow[t]{2}{*}{ Característica } & \multicolumn{2}{|c|}{$\begin{array}{l}\text { Atraso } \\
(n=99)\end{array}$} & \multicolumn{2}{|c|}{$\begin{array}{c}\text { Atualizado } \\
(n=414)\end{array}$} & \multirow[t]{2}{*}{ Valor de $p$} \\
\hline & $\mathrm{n}$ & $\%$ & $\mathrm{n}$ & $\%$ & \\
\hline \multicolumn{6}{|l|}{ Idade } \\
\hline 20 a 39 & 58 & 19,9 & 233 & 80,1 & 0,677 \\
\hline 40 a 59 & 41 & 18,5 & 181 & 81,5 & \\
\hline \multicolumn{6}{|l|}{ Cor da pele* } \\
\hline Parda/negra & 46 & 22,4 & 159 & 77,6 & 0,146 \\
\hline Branca/amarela & 53 & 17,3 & 254 & 82,7 & \\
\hline \multicolumn{6}{|l|}{ Estado conjugal } \\
\hline Sem companheiro & 35 & 24,3 & 109 & 75,7 & 0,073 \\
\hline Com companheiro & 64 & 17,3 & 305 & 82,7 & \\
\hline \multicolumn{6}{|l|}{ Trabalho fora de casa } \\
\hline Não & 71 & 22,4 & 246 & 77,6 & 0,024 \\
\hline Sim & 28 & 14,3 & 168 & 85,7 & \\
\hline \multicolumn{6}{|l|}{ Classe econômica } \\
\hline $\mathrm{D} / \mathrm{E}$ & 59 & 24,9 & 178 & 75,1 & 0,002 \\
\hline C & 32 & 17,5 & 151 & 82,5 & \\
\hline$A / B$ & 8 & 8,6 & 85 & 91,4 & \\
\hline \multicolumn{6}{|l|}{ Utilização da UBS } \\
\hline Não & 21 & 18,4 & 93 & 81,6 & 0,788 \\
\hline Sim & 78 & 19,5 & 321 & 80,5 & \\
\hline
\end{tabular}

*Excluído um caso com cor de pele sem anotação entre as mulheres com exame em dia.

\section{Discussão}

Este estudo mostra boa cobertura geral do exame Papanicolaou nas áreas pesquisadas, sendo atingida, na média, a meta estabelecida pela Organização Mundial da Saúde de, no mínimo, 80\% de cobertura do exame ${ }^{1}$, embora esta, em uma das UBS, tenha sido de apenas $71,5 \%$. Apesar de diferenças metodológicas, estudos recentes mostraram as seguintes porcentagens de coberturas referentes aos três anos anteriores às entrevistas no Brasil e em países da América do Sul: 77,3\% no município de São Paulo em $2000^{12}$, 68,8\% no município de Pelotas em $2002^{20}$, 30,5\% em uma localidade da Argentina em $2003^{21}$ e $66 \%$ no Chile em $1996^{8}$. Nos Estados Unidos, no entanto, essa proporção é mais elevada: $83 \%$ entre mulheres com idade igual ou superior a 18 anos entrevistadas em $2000^{22}$.

Apesar da boa cobertura encontrada, e de todas as mulheres residirem em áreas cobertas pela estratégia Saúde da Família, a qual tem se mostrado capaz de reduzir as iniqüidades sociais no acesso aos serviços de saúde ${ }^{14}$, confirma-se, neste estudo, a desigualdade na utilização do exame. Em estudo realizado em Pelotas (RS), observou-se maior percentual de atraso entre mulheres de classes econômicas menos favorecidas, não brancas e com menor escolaridade ${ }^{20}$. Tais achados reforçam a necessidade de os profissionais e gestores de saúde estarem atentos às dificulda- 
des de acesso e de utilização dos serviços por essa parcela da população, especialmente sob modalidade de atenção que pressupõe ênfase na promoção da saúde e na prevenção de agravos, como a da Saúde da Família.

No presente estudo, chama a atenção o maior percentual de atraso entre as mulheres que não trabalham fora de casa. Há relato na literatura ${ }^{21}$ de que as mulheres que trabalham fora de casa apresentam proporções mais elevadas de atitude adequada em face do exame Papanicolaou, ou seja, para elas, há necessidade de fazer o exame periodicamente. Como apontam os autores desse estudo argentino $^{21}$, questões de gênero, possivelmente, permeiam essa associação, fazendo com que mulheres que trabalham exclusivamente em casa tenham menos autonomia para tomar decisões relativas à sua saúde. Outra possibilidade é a de que mulheres que trabalham fora de casa têm maior acesso a informações nos contatos com outras trabalhadoras ou empregadoras, o que pode estimular práticas preventivas de saúde.

Foram constatados, neste estudo, percentuais semelhantes de não-realização do exame nos últimos três anos nas faixas etárias de 20-39 e de 40-59 anos. No entanto, alguns trabalhos apontam que deve ser dada atenção especial às mulheres à medida que estas se afastam do periodo fértil ${ }^{1,23}$. Observa-se que nos países em desenvolvimento a prática do exame preventivo do câncer de colo de útero está muitas vezes relacionada à maternidade. Sendo assim, a cobertura adequada não se estende a todas as mulheres que têm risco de desenvolver a doença ${ }^{1}$. Além disso, há maior risco de incidência de câncer uterino com o aumento da idade, como revela estudo conduzido nos Estados Unidos da América, em que as proporções de exames de citologia oncótica alterados foram maiores nas faixas etárias mais jovens, porém as taxas de câncer invasivo aumentaram com a idade, atingindo valores máximos entre 50 a 64 anos $^{23}$.

Foi constatada, nesta investigação, maior proporção de desconhecimento a respeito da data de agendamento do próximo exame entre as mulheres que referiram sua última coleta nas UBS, quando comparadas àquelas que referiram coleta em locais que prestam atendimento privado ou por convênios médicos. Dados semelhantes foram observados no municipio de Pelotas, onde mulheres que tiveram a última consulta em clínica particular ou em locais que atendiam por convênio médico sabiam mais sobre o exame quando comparadas às que haviam procurado o serviço público de saúde ${ }^{20}$. Mesmo controlando o efeito da classe econômica na possibilidade de conhecimento, foi menor a proporção de mulheres que sabiam informar a data aproximada do próximo exame entre as usuárias das UBS de Londrina, o que requer a implementação de ações específicas visando à melhoria na transmissão dessa informação nesses serviços.

Quanto aos motivos para a adesão ao último exame, os resultados deste estudo diferem dos apresentados em São Paulo ${ }^{12}$. O principal motivo referido pelas entrevistadas desta pesquisa foi a "rotina do programa" oferecido pela UBS, ao passo que em São Paulo foi a demanda espontânea. Embora diferenças metodológicas não possam ser descartadas entre os possiveis motivos para essas realidades diversas, essa diferença pode indicar maior adesão da população pesquisada, no presente estudo, ao programa de prevenção do câncer de colo uterino. Dentre os prováveis motivos para esta adesão podem ser citados a distribuição geográfica das UBS (próximas à população, com tempo médio de deslocamento de 16 minutos), a forma de organização do trabalho e a atuação das equipes de saúde da família, em especial dos agentes comunitários de saúde, pelo contato direto e freqüente com a população de sua área de atuação.

Os motivos citados na literatura para a nãoadesão ao exame são bastante variados e alguns deles semelhantes aos achados deste estudo. A ausência de sintomas e a vergonha foram, por exemplo, apontados em estudo realizado na Argentina $^{21}$, como, respectivamente o segundo e o quinto principais motivos para a não-adesão ao exame. Em São Paulo ${ }^{12}$, a ausência de sintomas ginecológicos foi a principal razão relatada pelas mulheres entrevistadas (45\%) para não terem se submetido ao exame, seguida por sentimentos de medo do exame ou do diagnóstico, vergonha ou desconforto físico $(32,5 \%)$. Diante dos motivos expostos para a não-adesão ao exame, faz-se necessário o desenvolvimento de estratégias a serem adotadas pelas equipes de saúde na tentativa de minimizar este problema. Os programas de saúde precisam ser adaptados à realidade local e serem sensiveis às múltiplas barreiras que as mulheres vivenciam no acesso aos serviços ${ }^{1,12}$.

Cabe destacar que o presente estudo concentrou-se na ótica das mulheres sobre os motivos para adesão ou não ao exame Papanicolaou. Sabe-se, no entanto, que o sucesso de um programa de rastreamento deste tipo depende de múltiplos fatores, em especial dos ligados aos aspectos organizacionais dos serviços de saúde. Motivos de não-adesão apontados por algumas mulheres (dificuldades no agendamento do exame e de relacionamento profissional-cliente) poderiam ser classificados como relacionados a tais aspectos, os quais incluem a disponibilidade de serviços, ma- 
teriais e recursos humanos adequados à atenção necessária, protocolos de atendimento eficazes, acessibilidade aos serviços, comunicação efetiva profissional-cliente, além de garantia de tratamento nos casos necessários, entre outros ${ }^{10,24}$. Estudo realizado nos Estados Unidos da América, com mulheres imigrantes vietnamitas, identificou maiores taxas de adesão ao Papanicolaou entre as que sabiam que o exame é necessário mesmo nos casos sem sintomatologia, entre as que haviam recebido aconselhamento médico para realizar o exame ou entre aquelas que haviam se sentido à vontade para conversar com o profissional e solicitar o exame ${ }^{24}$, reforçando a importância da boa relação profissional-cliente e da comunicação efetiva, as quais possibilitam ampliar o conhecimento a respeito da importância do exame entre as usuárias dos serviços de saúde.

Por outro lado, modificações introduzidas nos programas de rastreamento, em geral sem aumento expressivo de custos, podem ser fundamentais para elevar a adesão das mulheres, tais como relatam trabalhos desenvolvidos em outros países, como, por exemplo, no Chile, onde a cobertura do exame na faixa etária de maior risco elevou-se expressivamente após reorganização do programa nacional ${ }^{8}$, e na Suécia, onde também ocorreu aumento da cobertura do exame e redução do número de exames desnecessários ${ }^{7}$.

Em relação a estratégias possiveis de serem adotadas pelos serviços locais de saúde, estudo de revisão sistemática reporta que mudanças no processo de atenção, com a introdução da oferta do exame no mesmo dia em que a mulher comparece ao serviço, foi a intervenção mais efetiva no aumento da proporção de exames realizados $(32,7 \%)$, embora sucessos também tenham sido obtidos em estratégias baseadas em avisos por carta ou telefone às mulheres com exames atrasados, implantação de registros diários nos serviços para identificar mulheres com o exame em atraso, entre outras ${ }^{25}$. Em Londrina, cuja população conta com alta cobertura da Saúde da Família, poder-se-ia considerar a necessidade e pertinência de implementar ações protocolizadas de identificação das mulheres com o exame atrasado, proporcionando busca ativa e agendamento facilitado do exame. Além disso, o próprio cadastro da família, o qual é periodicamente renovado nas visitas dos agentes comunitários, poderia incluir pergunta a respeito do exame de citologia oncótica das mulheres da faixa etária de maior risco, identificando, também, aquelas que nunca realizaram o exame.

Apesar de os dados obtidos no presente estudo serem limitados a áreas de apenas cinco UBS do município de Londrina, os resultados podem ser úteis na reorientação de ações do Serviço Municipal de Saúde, partindo-se da definição do perfil das mulheres que estão em atraso com o exame e dos fatores que contribuem para o não comparecimento das mulheres para coleta, pois as cinco unidades pesquisadas possuem perfil muito semelhante ao encontrado nas demais unidades básicas de saúde que compõem o Serviço.

A atuação das equipes de saúde da família no municipio de Londrina pode ter tido influência na boa cobertura do exame Papanicolaou. No entanto, são necessárias ações que visem obter maior adesão das mulheres em atraso com o exame, em especial daquelas com piores condições financeiras e que não trabalham fora de casa. Também é importante maior empenho dos profissionais atuantes nas UBS para que as mulheres sejam mais bem informadas em relação à data de agendamento do próximo exame, visando sua realização em adequada periodicidade.

\section{Referências}

1. World Health Organization. Cervical cancer screening in developing countries: report of a WHO consultation. Geneva; 2002.

2. Danaei G, Vander Hoorn S, Lopez AD, Murray CJ, Ezzati M; Comparative Risk Assessment Collaborating Group (Cancers). Causes of cancer in the world: comparative risk assessment of nine behavioural and environmental risk factors. Lancet. 2005;366(9499):1784-93.

3. Ministério da Saúde. DATASUS [homepage da Internet]. Informações em saúde. Brasília; 2006 [2006 Jan 11]. Disponivel em: http://www.datasus.gov.br.

4. Fonseca LAM, Ramacciotti AS, Eluf Neto J. Tendência da mortalidade por câncer de útero no Município de São Paulo. Cad Saúde Pública. 2004;20(1):136-42.

5. Ministério da Saúde. Secretaria de Atenção à Saúde. Instituto Nacional de Câncer [homepage na Internet]. Estimativa 2006: Incidência de câncer no Brasil. Rio de Janeiro; 2006 [2006 Jan 11]. Disponivel em: http://www.inca.gov.br/estimativa/2006/

6. Leal EAS, Leal Júnior OS, Guimarães MH, Vitoriano MN, Nascimento TL, Costa OL. Lesões precursoras do câncer de colo em mulheres adolescentes e adultas jovens do município de Rio Branco - Acre. Rev Bras Ginecol Obstet. 2003;25(2):81-6.

7. Nygard JF, Skare GB, Thoresen SO. The cervical cancer screening programme in Norway, 1992-2000: changes in Pap smear coverage and incidence of cervical cancer. J Med Screen. 2002;9(2):86-91. 
8. Sepulveda C, Prado R. Effective cervical cytology screening programmes in middle-income countries: the Chilean experience. Cancer Detect Prev. 2005;29(5):405-11.

9. Ministério da Saúde. Instituto Nacional de Câncer. Periodicidade de realização do exame preventivo do câncer do colo do útero: normas e recomendações do INCA. Rev Bras Cancerol. 2002;48(1):13-5.

10. Pinho AA, França Junior I. Prevenção do câncer de colo de útero: um modelo teórico para analisar o acesso e a utilização do teste de Papanicolaou. Rev Bras Saúde Matern Infant. 2003;3(1):95-112.

11. Martins LFL, Thuler LCS, Valente JG. Cobertura do exame de Papanicolaou no Brasil e seus determinantes: uma revisão sistemática da literatura. Rev Bras Ginecol Obstet. 2005;27(8):48592.

12. Pinho AA, França Junior I, Schraiber LB, D’Oliveira AFPL. Cobertura e motivos para a realização ou não do teste de Papanicolaou no Município de São Paulo. Cad Saúde Pública. 2003;19 Supl 2:S303-13.

13. Bradley J, Barone M, Mahe C, Lewis R, Luciani S. Delivering cervical cancer prevention services in low-resource settings. Int $J$ Gynaecol Obstet. 2005;89 Suppl 2:S21-9.

14. Goldbaum M, Gianini RJ, Novaes HMD, César CLG. Utilização de serviços de saúde em áreas cobertas pelo programa saúde da família (Qualis) no Município de São Paulo. Rev Saúde Pública. 2005;39(1):90-9.

15. Universidade Estadual de Londrina (UEL). Centro de Ciências da Saúde. Práticas de interação ensinoserviço-comunidade. Londrina: Ed. UEL; 2005.

16. Silva AMR, Oliveira MSM, Nunes EFPA, Torres ZF. A unidade de saúde e seu território. In: Andrade SM, Soares DA, Cordoni Junior L, organizadores. Bases da saúde coletiva. Londrina: UEL; 2001.
17. Associação Brasileira de Empresas de Pesquisa (ABEP). Critério de classificação econômica Brasil. São Paulo: ABEP; 2003.

18. Dean AG, Dean JA, Coulombier D, Brendel KA, Smith $\mathrm{DC}$, Burten $\mathrm{AH}$, et al. Epi Info, version 6: a wordprocessing, database, and statistics program for public health on IBM-compatible microcomputers. Atlanta: Centers for Disease Control and Prevention; 1995.

19. Soares JF, Siqueira AL. Introdução à estatística médica. 2a ed. Belo Horizonte: Coopmed; 2002.

20. Quadros CAT, Victora CG, Dias da Costa JS. Coverage and focus of a cervical cancer prevention program in southern Brazil. Rev Panam Salud Pública. 2004;16(4):223-32.

21. Gamarra CJ, Paz EPA, Griep RH. Conhecimentos, atitudes e prática do exame de Papanicolaou entre mulheres argentinas. Rev Saúde Pública. 2005;39(2):270-6.

22. Hewitt M, Devesa SS, Breen N. Cervical cancer screening among U.S. women: analyses of the 2000 National Health Interview Survey. Prev Med. 2004;39(2):270-8.

23. Bernard VB, Eheman CR, Lawson HW, Blackman DK, Anderson C, Helsel W, et al. Cervical screening in the National Breast and Cervical Cancer Early Detection Program, 1995-2001. Obstet Gynecol. 2004;103(3):564-71.

24. Taylor VM, Yasui Y, Burke N, Nguyen T, Acorda E, Thai H, Qu P, Jackson JC. Pap testing adherence among Vietnamese American women. Cancer Epidemiol Biomarkers Prev. 2004;13(4):613-9.

25. Yabroff KR, Mangan P, Mandelblatt J. Effectiveness of interventions to increase Papanicolaou smear use. J Am Board Fam Pract. 2003;16(3):188-203. 